
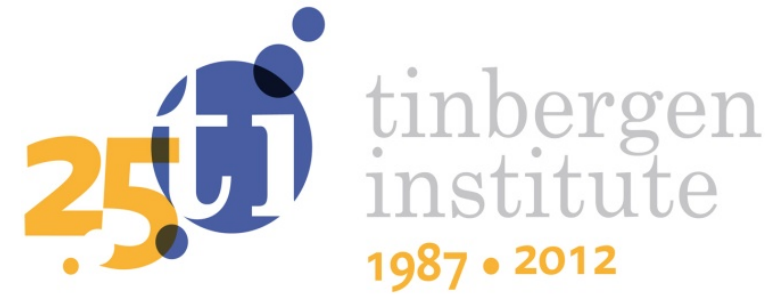

\title{
A Strategic Implementation of the Average Tree Solution for Cycle-Free Graph Games
}

René van den Brink

Gerard van der Laan

Nigel Moes

Faculty of Economics and Business Administration, VU University Amsterdam, and Tinbergen Institute. 
Tinbergen Institute is the graduate school and research institute in economics of Erasmus University Rotterdam, the University of Amsterdam and VU University Amsterdam.

More TI discussion papers can be downloaded at http://www.tinbergen.nl

Tinbergen Institute has two locations:

Tinbergen Institute Amsterdam

Gustav Mahlerplein 117

1082 MS Amsterdam

The Netherlands

Tel.: +31(0)205251600

Tinbergen Institute Rotterdam

Burg. Oudlaan 50

3062 PA Rotterdam

The Netherlands

Tel.: +31(0)10 4088900

Fax: $+31(0) 104089031$

Duisenberg school of finance is a collaboration of the Dutch financial sector and universities, with the ambition to support innovative research and offer top quality academic education in core areas of finance.

DSF research papers can be downloaded at: http://www.dsf.nl/

Duisenberg school of finance

Gustav Mahlerplein 117

1082 MS Amsterdam

The Netherlands

Tel.: +31(0)20 5258579 


\title{
A Strategic Implementation of the Average Tree Solution for Cycle-Free Graph Games*
}

\author{
René van den Brink ${ }^{\dagger}$ Gerard van der Laan ${ }^{\ddagger}$ Nigel Moes $^{\S}$
}

May 7, 2012

*This research is financially supported by Netherlands Organization for Scientific Research NWO grant 400-07-159.

${ }^{\dagger}$ J.R. van den Brink, Department of Econometrics and Tinbergen Institute, VU University Amsterdam, De Boelelaan 1105, 1081 HV Amsterdam, the Netherlands. E-mail: jrbrink@feweb.vu.nl

${ }^{\ddagger}$ G. van der Laan, Department of Econometrics and Tinbergen Institute, VU University Amsterdam, De Boelelaan 1105, 1081 HV Amsterdam, the Netherlands. E-mail: glaan@feweb.vu.nl

$\S_{N}$. Moes, Department of Econometrics and Tinbergen Institute, VU University Amsterdam, De Boelelaan 1105, 1081 HV Amsterdam, the Netherlands. E-mail: nmoes@feweb.vu.nl 


\begin{abstract}
In this note we provide a strategic implementation of the average tree solution for zeromonotonic cycle-free graph games. That is, we propose a non-cooperative mechanism of which the unique subgame perfect equilibrium payoffs correspond to the average hierarchical outcome of the game. This mechanism takes into account that a player is only able to communicate with other players (i.e., to make proposals about a division of the surplus of cooperation) when they are connected in the graph.
\end{abstract}

Keywords: implementation, cycle-free graph game, tree game, hierarchical outcome, average tree solution, weighted hierarchical outcome.

JEL codes: C71, C72 


\section{Introduction}

Pérez-Castrillo and Wettstein (2001) provide a strategic implementation of the Shapley value (Shapley, 1953) for zero-monotonic transferable utility (TU) games. That is, they propose a non-cooperative extensive form game of which the unique subgame perfect equilibrium (SPE) payoffs correspond to the Shapley value payoffs of the cooperative game. Following PérezCastrillo and Wettstein (2001), several authors have suggested similar mechanisms for various models and solutions. ${ }^{1}$

In this note we adapt the mechanism of Pérez-Castrillo and Wettstein (2001) to obtain an implementation of the average tree solution for zero-monotonic cycle-free graph games with transferable utility. Cooperative TU-games with a (communication) graph structure, or graph games, were introduced by Myerson (1977) and consist of a cooperative game with transferable utility, or shortly TU-game, and a graph on the set of players in the game. The players in a graph game are only able to cooperate (communicate) when they are connected in the graph. A graph therefore provides a cooperation structure on the set of players in a TU-game, that restricts their cooperation possibilities. Myerson (1977) also extended the Shapley value for TU-games to the class of graph games. This value has become known as the Myerson value for graph games.

A graph game is cycle-free if the graph does not contain any cycle. For this class of games Herings, van der Laan, Talman (2008) proposed the average tree solution, which assigns to every cycle-free graph game the average of all hierarchical outcomes, introduced in Demange (2004). They provide an axiomatic characterization using the well-known component efficiency and introducing component fairness (saying that deleting a link between two players yields for both resulting components, that is the subgraphs remaining after deleting the link, the same average change in payoff) as an alternative to fairness of the Myerson value (saying that deleting a link between two players yields the same change in payoff for both players incident with the link).

The goal of this note is to adapt the mechanism of Pérez-Castrillo and Wettstein (2001) to implement the average hierarchical outcome for tree games, i.e. connected, cycle-free graph games. Similar as in their paper, in the first stage of the mechanism developed here each player makes a bid to all other players and the player with the highest net bid pays its bids. Then, in the second stage this player makes a proposal on the division of the total payoff to all other players. If all others accept, the game ends and each player gets the proposed amount. If the proposal is rejected, the proposer leaves. Different from Pérez-Castrillo and Wettstein (2001),

\footnotetext{
${ }^{1}$ For example, efficient outcomes in a general social choice network in Pérez-Castrillo and Wettstein (2002); the Owen value for games with coalition structures in Vidal-Puga and Bergantiños (2003); efficient outcomes in local public goods environments in Mutuswami, Pérez-Castrillo and Wettstein (2004); efficient networks in Pérez-Castrillo and Wettstein (2005); a Shapley-type outcome for games with externalities in Macho-Stadler, Pérez-Castrillo and Wettstein (2006); the position value, Myerson value and the component-wise egalitarian value for games with graph structure in Slikker (2007); a generalized bidding approach for several values in Ju and Wettstein (2009); and the egalitarian and discounted Shapley values in van den Brink and Funaki (2010) and van den Brink, Funaki and Ju (2011).
} 
now the communication graph is taken into account and each of the neighbors of the original proposer becomes a proposer and makes a proposal about the division of the total payoff to the players in its component of the subgraph on the remaining players. If the proposal in a particular component is accepted by all the players in the component, all these players get the proposed amount and leave the mechanism. If the proposal in a particular component is rejected, the proposer leaves and its neighbors become proposer in their own (sub)components. This continues until either all players in all components have accepted their proposals or no players are left.

What should be observed is that within a communication graph structure a player is only able to communicate with other players when they are connected in the graph. This immediately highlights a main difference between the mechanism in this note and that of Pérez-Castrillo and Wettstein (2001), in that in their mechanism there is bidding on who will be the new proposer each time a proposal is rejected, whereas in our mechanism, once a first proposer has been established, the order in which the players are allowed to make proposals is fixed by the graph structure. This also might result in multiple proposers when the graph on the remaining players consists of several components. Nevertheless, the mechanism has similar desirable features as the mechanism of Pérez-Castrillo and Wettstein (2001). Namely, (i) the players receive their average hierarchical outcome in every SPE outcome, not only in expectation, (ii) there is no a priori randomization that imposes an order on the moves of the players in the mechanism, (iii) the mechanism is finite and (iv) the equilibrium strategies are simple, moreover they are unique when the game is strictly zero-monotonic.

This note is organized as follows. In Section 2 we give some preliminaries on TU-games and (communication) graph games. Before presenting our main result, in Section 3 we discuss a mechanism that implements the marginal vectors of a TU-game (without graph) and give a mechanism that implements the hierarchical outcomes of a tree game. These findings are then used in Section 4 to present the main mechanism of this note that implements the average hierarchical outcome on the class of zero-monotonic tree games. ${ }^{2}$

\section{Communication graph games}

A cooperative game with transferable utility, shortly a $T U$-game, is a pair $(N, v)$, where $N \subset \mathbb{N}$ is a finite set of $n=|N|$ players and $v: 2^{N} \rightarrow \mathbb{R}$ is a characteristic function on $N$ such that $v(\emptyset)=0$. A subset $S \subseteq N, S \neq \emptyset$, is called a coalition. For any coalition $S, v(S)$ displays the worth of that coalition, which can be interpreted as the wealth, measured in units of transferable utility, that the members of coalition $S$ are able to divide among themselves when they decide to cooperate. The collection of all TU-games is denoted by $\mathcal{G}$. For $S \subset N$, the TU-game $\left(S, v_{S}\right)$ with $v_{S}$ given by $v_{S}(T)=v(T)$ for every $T \subseteq S$, is the subgame restricted to $S$. We sometimes denote $\left(S, v_{S}\right)$ by $(S, v)$. A TU-game $(N, v)$ is zero-monotonic if $v(S)+v(\{i\}) \leq v(S \cup\{i\})$ for

\footnotetext{
${ }^{2}$ Although stated for tree games for notational convenience, the implementations work for any cycle-free graph game.
} 
every $i \in N$ and $S \subseteq N \backslash\{i\}$. A payoff vector for $(N, v) \in \mathcal{G}$ is a vector $x \in \mathbb{R}^{N}$, assigning a payoff $x_{i}$ to every $i \in N$. A (single-valued) solution or value on $\mathcal{G}$ is a function $f$ on $\mathcal{G}$ that assigns a payoff vector $f(N, v) \in \mathbb{R}^{N}$ to every $(N, v) \in \mathcal{G}$. An ordering on $N \subset \mathbb{N}$ is a one-to-one mapping $\pi: N \rightarrow\{1, \ldots, n\}$ assigning a rank number $\pi(k) \in\{1, \ldots, n\}$ to every player $k \in N{ }^{3}$ We denote the collection of all orderings on $N$ by $\Pi(N)$. Given an ordering $\pi \in \Pi(N)$, we define $\pi^{i}=\{j \in N \mid \pi(j) \leq \pi(i)\}$ as the set of all players $j$ with rank number $\pi(j)$ at most equal to rank number $\pi(i)$ of player $i$, including $i$ itself. For $\pi \in \Pi(N)$, the marginal vector $m^{\pi}(N, v) \in \mathbb{R}^{N}$ of TU-game $(N, v)$ is given by $m_{i}^{\pi}(N, v)=v\left(\pi^{i}\right)-v\left(\pi^{i} \backslash\{i\}\right), i \in N$. The Shapley value (Shapley (1953)) assigns to every $(N, v) \in \mathcal{G}$ the average of all $|N|$ ! marginal (payoff) vectors $m^{\pi}(N, v), \pi \in \Pi(N)$.

A communication structure between players in a TU-game can be represented by an undirected graph being a pair $(N, L)$, where $N$ is a finite set of nodes (in this note equal to the set of players in a game) and $L \subseteq\{\{i, j\} \mid i \neq j, i, j \in N\}$ is a set of edges or links (which stands for the collection of communication links between the players). A sequence of $k$ different nodes $\left(i_{1}, \ldots, i_{k}\right)$ is called a path from $i_{1}$ to $i_{k}$ in the graph $(N, L)$ if $\left\{i_{h}, i_{h+1}\right\} \in L$ for $h=1, \ldots, k-1$. A coalition $S \subseteq N$ is connected in $(N, L)$ if for any $i, j \in S, i \neq j$, there is a path in $S$ from $i$ to $j$. A coalition $S$ is a component in $(N, L)$ if it is a maximally connected set, i.e., $S$ is connected and $S \cup\{j\}$ is not connected for all $j \in N \backslash S$. Given $(N, L)$, each $K \subseteq N$ induces a subgraph $(K, L(K))$, with $L(K)=\{\{i, j\} \in L \mid i, j \in K\}$ the set of links on $K$. For $K \subseteq N, C^{L}(K)$ denotes the collection of all components in $(K, L(K))$. A TU-game with (communication) graph structure, shortly a graph game, is a triple $(N, v, L)$, with $(N, v) \in \mathcal{G}$ a TU-game and $(N, L)$ an undirected graph. We denote the class of all graph games by $\mathcal{G} \mathcal{L}$. Myerson (1977) assumes that in a graph game $(N, v, L)$, a coalition $S \subseteq N$ can only cooperate and realize its worth $v(S)$ if $S$ is connected. Under this assumption, the (Myerson) restricted game $\left(N, v^{L}\right) \in \mathcal{G}$ is defined by $v^{L}(S)=\sum_{K \in C^{L}(S)} v(K)$ for every $S \subseteq N$, thus the worth $v^{L}(S)$ is equal to the sum of the worths of all components in $(S, L(S))$. The Myerson value assigns to every game $(N, v, L) \in \mathcal{G} \mathcal{L}$ the Shapley value of the restricted game $\left(N, v^{L}\right)$.

A path $\left(i_{1}, \ldots, i_{k}\right)$ is a cycle if $\left\{i_{k}, i_{1}\right\} \in L$. A graph $(N, L)$ is cycle-free if it does not contain a cycle. A graph $(N, L)$ is connected if $N$ is connected, and it is called a tree if it is cycle-free and connected. A graph game $(N, v, L)$ is a cycle-free graph game when $(N, L)$ is cycle-free, and it is a tree game when $(N, L)$ is a tree. For tree games, Demange (2004) introduced the so-called hierarchical outcomes. To define these outcomes, we first give the notion of a rooted tree. A pair $(N, D)$ is a directed graph or digraph with node set $N$ if $D \subseteq\{(i, j) \in N \times N \mid i \neq j\}$ is a collection of directed edges or arcs. A sequence of different nodes $\left(i_{1}, \ldots, i_{k}\right)$ is a directed path in $(N, D)$ if $\left(i_{h}, i_{h+1}\right) \in D$ for $h=1, \ldots, k-1$. A directed graph $(N, D)$ is a rooted tree if it has precisely $|N|-1$ arcs and there is a unique node $i \in N$, called the root, such that there is directed path from $i$ to every other node in $(N, D)$. Given an undirected tree $(N, L)$ and a player $i \in N$, let $\left(N, L^{i}\right)$ be the unique rooted tree with root $i$ induced by the tree $(N, L)$, i.e., $L^{i}=\{(j, h) \mid\{j, h\} \in L$, and $j$ is on the path from $i$ to $h\}$. In the rooted tree $\left(N, L^{i}\right)$, if

\footnotetext{
${ }^{3}$ In many studies $\pi$ is called a permutation on $N$, which implicitly assumes that $N=\{1, \ldots, n\}$. Since in this paper the player set can vary we refer to $\pi$ as an ordering.
} 
$(j, h) \in L^{i}$ then node $h$ is a successor, or follower, of $j$. We say that $h$ is a subordinate of $j$ if there is a directed path from $j$ to $h$. Given a tree $(N, L)$ and a node $i \in N, F_{j}^{i}$ denotes the set of followers of $j$ in $\left(N, L^{i}\right)$, and $S_{j}^{i}$ the set containing $j$ itself and all its subordinates in $\left(N, L^{i}\right)$. Notice that $S_{i}^{i}=N$ and that every set $S_{j}^{i}$ is connected in $(N, L)$. Now, for a tree game $(N, v, L)$ and some $i \in N$, the hierarchical outcome $h^{i}(N, v, L)$ is the marginal vector $m^{\pi}\left(N, v^{L}\right)$ of the (Myerson) restricted game for any ordering of the players that is consistent with the rooted tree $\left(N, L^{i}\right)$, i.e., if the path from $i$ to $g$ in $(N, L)$ contains $j$ then $\pi(g)<\pi(j)$. It follows that

$$
h_{j}^{i}(N, v, L)=v\left(S_{j}^{i}\right)-\sum_{h \in F_{j}^{i}} v\left(S_{h}^{i}\right), \quad j \in N .
$$

For tree games, Herings et al. (2008) introduce the so-called average tree solution as the value that assigns to every tree graph game the average of the $|N|$ hierarchical outcomes $h^{i}(N, v, L)$, $i \in N .{ }^{4}$ It thus assigns to every tree game $(N, v, L)$ the average hierarchical outcome given by

$$
A H O(N, v, L)=\frac{1}{|N|} \sum_{i \in N} h^{i}(N, v, L) .
$$

The purpose of the underlying paper is to implement this solution.

\section{An implementation of the hierarchical outcomes}

In this section we propose a non-cooperative extensive form game, called a mechanism, of which the unique SPE payoffs correspond to the payoffs in the hierarchical outcome $h^{i}(N, v, L)$, $i \in N$, of any tree game $(N, v, L)$ with $(N, v)$ zero-monotonic. Since the hierarchical outcome $h^{i}(N, v, L)$ is equal to the marginal vector of the restricted game $\left(N, v^{L}\right)$ for any ordering $\pi$ that is consistent with the rooted tree $\left(N, L^{i}\right)$, we first discuss a mechanism that for any given ordering $\pi \in \Pi(N)$ implements the marginal vector $m^{\pi}(N, v)$ of any zero-monotonic TU-game.

For a given ordering $\pi$ on $N$, let $\rho(k)=\pi^{-1}(n+1-k), k \in N$, be the player in $N$ with rank number $n+1-k$ in $\pi$. Each round of the mechanism that implements $m^{\pi}(N, v)$ consists of three stages. In Stage 1 of round 1, player $\rho(1$ ) (with rank number $n$ ) is assigned to be the proposer and makes a proposal about a division of $v(N)$. So, player $\rho(1)$ proposes a payoff to every other player in $N \backslash\{\rho(1)\}$. In Stage 2 of round 1, the players in $N \backslash\{\rho(1)\}$ sequentially either accept or reject the proposal. If all these players accept, then in Stage 3 the players in $N \backslash\{\rho(1)\}$ receive the proposed payoffs and $\rho(1)$ receives the remaining. Otherwise, $\rho(1)$ leaves the mechanism and receives $v(\{\rho(1)\})$, while the other players go to the next round to bargain over their worth $v(N \backslash\{\rho(1)\})$. This second round has the same three stages as the first round (but with $n-1$ players), and starts with player $\rho(2)$ (with rank number $n-1$ ) as proposer. In general, in round $t \in\{1, \ldots, n-1\}$ the mechanism proceeds with player $\rho(t)$ as proposer, until either at some round all remaining players accept the proposal or round $n=|N|$ is reached in

\footnotetext{
${ }^{4}$ In fact, in Herings et al. (2008) the average tree solution is defined on the class of cycle free graph games and the solution has been generalized to the class of all graph games in Herings, van der Laan, Talman and Yang (2010).
} 
which only one player is left who just receives its singleton worth. Note that, different from the mechanism in Pérez-Castrillo and Wettstein (2001), there is no bidding stage where the players bid to decide who becomes the proposer in this mechanism, because this is fully determined by the ordering $\pi$.

The next theorem states that, given $\pi \in \Pi(N)$, the above mechanism implements the marginal vector $m^{\pi}(N, v)$ if the TU-game $(N, v)$ is zero-monotonic. The proof of this theorem is similar to part of the proof of the main theorem in Pérez-Castrillo and Wettstein (2001) and is therefore given in Appendix A, together with a formal description of the mechanism.

Theorem 3.1 Let $(N, v) \in \mathcal{G}$ be a zero-monotonic $T U$-game and let $\pi \in \Pi(N)$ be given. The payoff vector in any SPE of the mechanism described above coincides with the payoff vector $m^{\pi}(N, v)$.

We now consider the tree game $(N, v, L)$ and modify the above mechanism to state a mechanism, called Mechanism A, that implements for a given $i \in N$ the hierarchical outcome $h^{i}(N, v, L)$ in SPE. Given a player $i \in N$, in the first round of Mechanism A the root $i$ in the rooted tree $\left(N, L^{i}\right)$ is the proposer in Stage 1 . If its proposal is accepted by all other players, the mechanism ends immediately and each player $j \neq i$ receives the proposed payoff while player $i$ receives the remainder. Otherwise, $i$ leaves the mechanism with its stand-alone worth $v(\{i\})$. Observe that then for each of the followers $j \in F_{i}^{i}$, the set $S_{j}^{i}$ (containing $j$ and its subordinates in $\left.\left(N, L^{i}\right)\right)$ is a component in the subgraph $(N \backslash\{i\}, L(N \backslash\{i\}))$ and is a directed subtree of $\left(N, L^{i}\right)$ with player $j$ as its root. If $S_{j}^{i}=\{j\}$ then $j$ also leaves the mechanism with its standalone worth. If $j$ has followers, then the set $S_{j}^{i}$ advances to the next round of the mechanism. In this next round the root $j$ of the rooted subtree on $S_{j}^{i}$ proposes a division of the worth $v\left(S_{j}^{i}\right)$ to its subordinates in $S_{j}^{i}$. If in some round a proposal of a player $j$ to its subordinates in $S_{j}^{i}$ is accepted, then all players in $S_{j}^{i}$ leave the mechanism with the proposed payoffs. If a proposal is rejected, then the proposer leaves the mechanism with its stand-alone worth and each of its followers becomes the root of the directed subtree on the set consisting of this follower and its subordinates in $\left(N, L^{i}\right)$. If such a follower has no subordinates, it leaves the mechanism with its stand-alone worth, otherwise it becomes the proposer to its subordinates in the next round.

Note that in this mechanism (i) in a round there can be multiple proposers, and (ii) in a round there can be at the same time several branches in which the proposal is accepted, and other branches in which the proposal is rejected. In a branch in which the proposal is rejected the players, except the top of the branch, go to the next round and bargain in several new subbranches. Since in each round at least one player leaves and the number of players is finite, the mechanism ends within at most $n-1$ rounds (if only two players are left, the mechanism ends whether the proposal is accepted or not). Also note that, similar as in the previous mechanism, there is no bidding stage to determine who is the proposer.

Mechanism A to implement the hierarchical outcome $h^{i}(N, v, L), i \in N$, of a tree game $(N, v, L)$.

Initiation: Set $P^{1}=\{i\}$, set $t=1$ and go to Stage 1 of round 1 . 
Stage 1: Every $j \in P^{t}$ proposes an offer $y_{k}^{j} \in \mathbb{R}$ to every subordinate $k \in S_{j}^{i} \backslash\{j\}$. Go to Stage 2.

Stage 2: For every $j \in P^{t}$, the subordinates $k \in S_{j}^{i} \backslash\{j\}$ of player $j$ sequentially either accept or reject the offer $y_{k}^{j}$. If all subordinates accept, then the proposal of $j$ is accepted; otherwise the proposal of $j$ is rejected. Go to Stage 3.

Stage 3: Consider every $j \in P^{t}$. If the proposal of $j$ is accepted, then each subordinate $k \in S_{j}^{i} \backslash\{j\}$ receives $y_{k}^{j}$, player $j$ receives $v\left(S_{j}^{i}\right)-\sum_{k \in S_{j}^{i} \backslash\{j\}} y_{k}^{j}$ and the players in $S_{j}^{i}$ (that is $j$ and all its subordinates) leave the mechanism. If the proposal of $j$ is rejected, then player $j$ leaves the mechanism and obtains $v(\{j\})$. Set $R^{t}=\left\{j \in P^{t} \mid\right.$ proposal of $j$ is rejected $\}, H^{t}=\bigcup_{j \in R^{t}} F_{j}^{i}$, and $O^{t}=\left\{j \in H^{t} \mid F_{j}^{i}=\emptyset\right\}$. If $j \in O^{t}$, then $j$ receives $v(\{j\})$ and leaves the mechanism. Set $P^{t+1}=H^{t} \backslash O^{t}$ as the set of proposers in the next round. If $P^{t+1}=\emptyset$, no players are left and the mechanism stops. Otherwise, set $t$ equal to $t+1$ and go to Stage 1 of the next round.

The next theorem states that, given $i \in N$, Mechanism A implements the hierarchical outcome $h^{i}(N, v, L)$ if $(N, v)$ is zero-monotonic. The proof follows from the fact that the hierarchical outcome $h^{i}(N, v, L)$ is a marginal vector $m^{\pi}(N, v)$ of the restricted game $\left(N, v^{L}\right)$ and that Mechanism A is identical to the mechanism of Theorem 3.1 for every $\pi$ that is consistent with $\left(N, L^{i}\right)$, except that in a round there can be multiple proposers. ${ }^{5}$ The proof is given in Appendix B.

Theorem 3.2 Let $(N, v, L) \in \mathcal{G} \mathcal{L}$ be a tree game with $(N, v)$ zero-monotonic and let $i \in N$ be given. The payoff vector in any SPE of Mechanism A coincides with the payoff vector $h^{i}(N, v, L)$.

\section{Implementation of the average hierarchical outcome}

In this section we use the results of Section 3 to present our main finding, namely a mechanism, called Mechanism B, that implements the average hierarchical outcome of a tree game $(N, v, L)$ with $(N, v) \in \mathcal{G}$ zero-monotonic. ${ }^{6}$ Similar as in Pérez-Castrillo and Wettstein (2001), there is a bidding procedure to decide who becomes the proposer, but only in the first round. In the bidding procedure every player $i \in N$ makes a $b i d b_{j}^{i}$ to every other player $j \in N \backslash\{i\}$, which is the amount that player $i$ will pay to each player $j$ when $i$ becomes the proposer. The net bid of player $i \in N$ is equal to $B^{i}=\sum_{j \in N \backslash\{i\}}\left(b_{j}^{i}-b_{i}^{j}\right)$. The proposer is then chosen with equal probability among the players that have the highest net bid. Since $h_{j}^{j}(N, v, L)=$

\footnotetext{
${ }^{5}$ This is similar as in Slikker (2007), in which the mechanism of Pérez-Castrillo and Wettstein (2001) is modified to implement the Myerson value of a graph game $(N, v, L)$.

${ }^{6}$ For simplicity we only consider tree games, but the result holds for any zero-monotonic cycle-free graph game if we let players bargain only within their own component.
} 


$$
\begin{aligned}
v(N)-\sum_{k \in F_{j}^{j}} v\left(S_{k}^{j}\right) & =v^{L}(N)-v^{L}(N \backslash\{j\}) \text { it follows that } \\
A H O_{j}(N, v, L) & =\frac{1}{|N|}\left(v^{L}(N)-v^{L}(N \backslash\{j\})\right)+\frac{1}{|N|} \sum_{i \in N \backslash\{j\}} h_{j}^{i}(N, v, L) .
\end{aligned}
$$

So, the average hierarchical outcome of a player $j \in N$ is the average of its marginal contribution to the grand coalition in the restricted game $\left(N, v^{L}\right)$ and its payoffs in the $|N|-1$ hierarchical outcome vectors $h^{i}(N, v, L), i \neq j$. Although there are similarities between formula (4.1) and the recursive formula of the Shapley value stated in Maschler and Owen (1989), formula (4.1) is not recursive. ${ }^{7}$ Consequently, for a mechanism to implement the average hierarchical outcome in SPE it is enough to have a single bidding procedure at the start of the mechanism. Using formula (4.1) we will show that in any SPE of Mechanism B the bids of the players are given by $b_{j}^{i}=A H O_{j}(N, v, L)-h_{j}^{i}(N, v, L), i, j \in N, i \neq j$. The winner of the bidding procedure pays its bids to the other players. Then the mechanism moves to the proposal procedure, which is Mechanism A, with the winner of the bidding procedure as the proposer in the first round.

\section{Mechanism B to implement the average hierarchical outcome $A H O(N, v, L)$ of a tree game $(N, v, L)$.}

Bidding Procedure: Each player $j \in N$ makes bids $b_{k}^{j} \in \mathbb{R}$ to every $k \in N \backslash\{j\}$. For each $j \in N$, let $B^{j}=\sum_{k \in N \backslash\{j\}}\left(b_{k}^{j}-b_{j}^{k}\right)$ be the net bid of player $j$. Select the player with the highest net bid and call this player $i$. In case of a non-unique maximizer, choose any of the bidders with the highest net bid to be player $i$ with equal probability. The selected player $i$ is the winner of the bidding procedure and pays every other player $j \in N \backslash\{i\}$ its bid $b_{j}^{i}$. So, each player $j \in N \backslash\{i\}$ receives $b_{j}^{i}$ and player $i$ receives $-\sum_{j \in N \backslash\{i\}} b_{j}^{i}$ at this stage. Go to the Proposal Procedure.

Proposal Procedure: Play Mechanism A, starting in Stage 1 of round 1 with $P^{1}=\{i\}$. Hence, the winner $i$ of the bidding procedure is the first proposer.

Notice that adding the bidding procedure to Mechanism A changes the final payoffs of the players. The payoff of a player is now equal to the sum of its payoff in the bidding procedure and its payoffs in the proposal procedure (Mechanism A). This gives the following total payoffs when player $i$ is the winner of the bidding procedure. When in round 1 the proposal of $i$ is accepted, its total payoff is $v(N)-\sum_{j \neq i}\left(y_{j}^{i}+b_{j}^{i}\right)$, where $y_{j}^{i}$ is its offer to player $j, j \neq i$, in the proposal procedure. In this case the players $j \neq i$ receive $y_{j}^{i}+b_{j}^{i}$. When the proposal is rejected, the payoff of $i$ is $v(\{i\})-\sum_{j \neq i} b_{j}^{i}$. When in some round $t>1$ the offers $y_{k}^{j}$ of a player $j \neq i$ to its subordinates are accepted, then such a player $j$ has final payoff $b_{j}^{i}+v\left(S_{j}^{i}\right)-\sum_{k \in S_{j}^{i} \backslash\{j\}} y_{k}^{j}$, otherwise its payoff is $b_{j}^{i}+v(\{j\})$. When in some round all subordinates $k$ of a proposer $j$ accept the offers $y_{k}^{j}$, then each subordinate $k$ has final payoff $b_{k}^{i}+y_{k}^{j}$. When a proposal of a

\footnotetext{
${ }^{7}$ The recursive formula for the Shapley value $S h$ is given by $S h_{j}(N, v)=\frac{1}{|N|}(v(N)-v(N \backslash\{j\}))+$ $\frac{1}{|N|} \sum_{i \in N \backslash\{j\}} S h_{j}\left(N \backslash\{i\}, v_{N \backslash\{i\}}\right), j \in N$. Using this formula Pérez-Castrillo and Wettstein (2001) prove that the SPE bids in their mechanism are given by $b_{j}^{i}=S h_{j}(N, v)-S h_{j}\left(N \backslash\{i\}, v_{N \backslash\{i\}}\right), i, j \in N, i \neq j$.
} 
player $j$ is rejected and for a follower $k$ of $j$ it holds that $F_{k}^{i}=\emptyset$, then the payoff to $k$ is equal to $b_{k}^{i}+v(\{k\})$.

Theorem 4.2 below states that Mechanism B implements the average hierarchical outcome $A H O(N, v, L)$ if the TU-game $(N, v)$ is zero-monotonic and the graph $(N, L)$ is a tree. To prove the theorem we use, besides Theorem 3.2, the following lemma.

Lemma 4.1 Let $(N, v, L) \in \mathcal{G L}$ be a tree game with $(N, v)$ zero-monotonic and, for each $i, j \in$ $N, j \neq i$, let $b_{j}^{i}=A H O_{j}(N, v, L)-h_{j}^{i}(N, v, L)$. Then, for every $i \in N, \sum_{j \in N \backslash\{i\}}\left(b_{j}^{i}-b_{i}^{j}\right)=0$.

Proof. For every $i \in N$ we have that

$$
\begin{aligned}
\sum_{j \in N \backslash\{i\}} & \left(b_{j}^{i}-b_{i}^{j}\right)=\sum_{j \in N \backslash\{i\}}\left(A H O_{j}(N, v, L)-h_{j}^{i}(N, v, L)-A H O_{i}(N, v, L)+h_{i}^{j}(N, v, L)\right) \\
& =\sum_{j \in N \backslash\{i\}} A H O_{j}(N, v, L)-(n-1) A H O_{i}(N, v, L)+\sum_{j \in N \backslash\{i\}}\left(h_{i}^{j}(N, v, L)-h_{j}^{i}(N, v, L)\right) \\
& =\sum_{j \in N} A H O_{j}(N, v, L)-n A H O_{i}(N, v, L)+\sum_{j \in N \backslash\{i\}}\left(h_{i}^{j}(N, v, L)-h_{j}^{i}(N, v, L)\right) \\
& =v(N)-\sum_{j \in N} h_{i}^{j}(N, v, L)+\sum_{j \in N \backslash\{i\}}\left(h_{i}^{j}(N, v, L)-h_{j}^{i}(N, v, L)\right) \\
& =v(N)-h_{i}^{i}(N, v, L)-\sum_{j \in N \backslash\{i\}} h_{j}^{i}(N, v, L)=v(N)-\sum_{j \in N} h_{j}^{i}(N, v, L)=0,
\end{aligned}
$$

where the fourth equality follows by efficiency and the definition of the average hierarchical outcome, and the last equality follows from efficiency of the hierarchical outcome.

Theorem 4.2 Let $(N, v, L) \in \mathcal{G} \mathcal{L}$ be a tree game with $(N, v)$ zero-monotonic. Then the payoff vector in any SPE of Mechanism B coincides with the average hierarchical outcome $A H O(N, v, L)$.

Proof. (i) We first show that the payoff vector $A H O(N, v, L)$ is indeed a SPE payoff vector of the mechanism. Consider the following strategy of a player $\ell \in N$ in Mechanism B. In the bidding procedure, player $\ell$ announces $b_{q}^{\ell}=A H O_{q}(N, v, L)-h_{q}^{\ell}(N, v, L)$ to every $q \in N \backslash\{\ell\}$. When player $i$ is the winner of the bidding procedure and $j \in P^{t}$ is a proposer in some round $t \geq 1$ of the proposal procedure, then $j$ proposes in Stage 1 the hierarchical outcome $y_{k}^{j}=h_{k}^{i}(N, v, L)$ to every $k$ in its set of subordinates $S_{j}^{i} \backslash\{j\}$. In Stage 2 every subordinate $k$ of a proposer $j$ accepts any offer at least equal to $h_{k}^{i}(N, v, L)$ and rejects any offer strictly smaller than $h_{k}^{i}(N, v, L)$.

Clearly, following these strategies the proposal procedure ends in round 1 with payoffs $h_{k}^{i}(N, v, L), k \in N$, when $i$ is the winner of the bidding procedure. So, the total payoff to the winner $i$ in this strategy profile is equal to

$$
h_{i}^{i}(N, v, L)-\sum_{j \neq i} b_{j}^{i}=h_{i}^{i}(N, v, L)-\sum_{j \neq i}\left(A H O_{j}(N, v, L)-h_{j}^{i}(N, v, L)\right)
$$




$$
=\sum_{j \in N} h_{j}^{i}(N, v, L)-\sum_{j \neq i} A H O_{j}(N, v, L)=v(N)-\sum_{j \neq i} A H O_{j}(N, v, L)=A H O_{i}(N, v, L)
$$

where the third and fourth equality follow from efficiency of the hierarchical outcome. For player $j \neq i$ the total payoff is equal to

$$
h_{j}^{i}(N, v, L)+b_{j}^{i}=h_{j}^{i}(N, v, L)+A H O_{j}(N, v, L)-h_{j}^{i}(N, v, L)=A H O_{j}(N, v, L) .
$$

Hence, the above strategy profile indeed result in the payoff vector $A H O(N, v, L)$.

Next we show that these strategies are indeed SPE strategies. By Theorem 3.2 the strategy profile is a SPE profile in the subgame that starts in Stage 1 of round 1 of the proposal procedure (and all subsequent subgames). We therefore only have to consider the (sub)game that starts in the bidding procedure of Mechanism B.

Suppose that every player $i \neq k$ makes bids $b_{j}^{i}=A H O_{j}(N, v, L)-h_{j}^{i}(N, v, L), j \in N \backslash\{i\}$, and that some player $k$ deviates and makes bids $\left(\bar{b}_{j}^{k}\right)_{j \in N \backslash\{k\}}$ with $\bar{b}_{j}^{k} \neq A H O_{j}(N, v, L)-h_{j}^{k}(N, v, L)$ for at least one $j \neq k$. By Lemma 4.1 we know that in the strategy profile discussed above the net bid $B^{i}=\sum_{j \in N \backslash\{i\}}\left(b_{j}^{i}-b_{i}^{j}\right)=0$ for every $i \in N$. When for the deviating player $k$ the corresponding net bid $\bar{B}^{k}=\sum_{j \in N \backslash\{k\}}\left(\bar{b}_{j}^{k}-b_{k}^{j}\right)<B^{k}$, it follows that some other player $i \neq k$ wins the bidding. Then $i$ pays its bids $b_{j}^{i}$ in the bidding procedure and $h^{i}(N, v, L)$ in the proposal procedure, so deviating does not change the payoffs. When $\bar{B}^{k}=B^{k}$, then there must be a player $i \neq k$ who wins the bidding, and again this does not change the payoff of player $k$. Thus, we only have to consider the case that $\bar{B}^{k}>0=B^{k}$, i.e. $\sum_{j \in N \backslash\{k\}} \bar{b}_{j}^{k}>\sum_{j \in N \backslash\{k\}} b_{j}^{k}$, and thus the deviating player $k$ wins the bidding procedure. In the proposal procedure player $k$ makes offers $h_{j}^{k}(N, v, L)$ (which are its SPE offers in the proposal subgame by Theorem 3.2), and these offers are accepted. Hence $k$ 's final payoff is $v(N)-\sum_{j \in N \backslash\{k\}} h_{j}^{k}(N, v, L)-\sum_{j \in N \backslash\{k\}} \bar{b}_{j}^{k}=$ $h_{k}^{k}(N, v, L)-\sum_{j \in N \backslash\{k\}} \bar{b}_{j}^{k}<h_{k}^{k}(N, v, L)-\sum_{j \in N \backslash\{k\}} b_{j}^{k}=A H O_{k}(N, v, L)$. This means that the above strategy profile is indeed a SPE profile and it follows that $A H O(N, v, L)$ is a SPE payoff vector of Mechanism B.

(ii) It remains to prove that any SPE of Mechanism B yields the average hierarchical outcome. Similar as in Pérez-Castrillo and Wettstein (2001), it follows that all net bids are zero in any SPE (i.e. $B^{i}=0$ for all $i \in N$ ) and, consequently, in any SPE each player's payoff must be the same, regardless of the winner of the bidding procedure. ${ }^{8}$

By Theorem 3.2, $h^{k}(N, v, L)$ is implemented in the proposal procedure when $k$ wins the bidding procedure. Now consider some player $i$. If $i$ itself is the winner, then its final payoff is $x_{i}^{i}=h_{i}^{i}(N, v, L)-\sum_{j \in N \backslash\{i\}} b_{j}^{i}$ and when some player $j \neq i$ is the winner $i$ gets final payoff $x_{i}^{j}=h_{i}^{j}(N, v, L)+b_{i}^{j}$. Summing over all $k \in N$ gives

$\sum_{k \in N} x_{i}^{k}=\sum_{k \in N} h_{i}^{k}(N, v, L)+\sum_{k \in N \backslash\{i\}}\left(b_{i}^{k}-b_{k}^{i}\right)=|N| A H O_{i}(N, v, L)-B^{i}=|N| A H O_{i}(N, v, L)$.

Since $i$ is indifferent to the identity of the winner, it holds that $x_{i}^{h}=x_{i}^{k}$ for all $h, k \in N$ and thus $x_{i}^{j}=A H O_{i}(N, v, L)$ for all $j \in N$.

${ }^{8}$ This follows in the same way as Claims (c) and (d) in the proof of Theorem 1 in Pérez-Castrillo and Wettstein (2001). 
Notice that, as in the implementation of the Shapley value of Pérez-Castrillo and Wettstein (2001), in any SPE of Mechanism B all net bids are equal and therefore the choice of a random proposer is the outcome of the strategic bidding process. Further, it also holds that Mechanism $\mathrm{B}$ has a unique $\mathrm{SPE}$ when the game $(N, v) \in \mathcal{G}$ is strictly zero-monotonic. In that case each player $k \in N$ makes bids $b_{j}^{k}=A H O_{j}(N, v, L)-h_{j}^{k}(N, v, L)$ to the players $j \in N \backslash\{k\}$ in the bidding procedure; some player $i$ is randomly chosen to be the winner and becomes the proposer in round 1 of the proposal procedure. This player proposes $y_{j}^{i}=h_{j}^{i}(N, v, L)$ to every player $j \in N \backslash\{i\}$ and every player $j \in N \backslash\{i\}$ accepts the proposal.

In a similar way as Pérez-Castrillo and Wettstein (2001) extend their implementation of the Shapley value to weighted Shapley values, the result of Theorem 4.2 can easily be generalized to obtain an implementation of the weighted hierarchical outcomes. ${ }^{9}$ Given a vector of weights $\alpha \in \mathbb{R}_{+}^{N}$ with $\sum_{i \in N} \alpha_{i}=1$, the weighted hierarchical outcome $W H O^{\alpha}$ on the class of tree games is given by

$$
W H O^{\alpha}(N, v, L)=\sum_{i \in N} \alpha_{i} h^{i}(N, v, L)
$$

In particular, $\operatorname{WHO}^{\alpha}(N, v, L)=A H O(N, v, L)$ if $\alpha_{i}=\frac{1}{|N|}$ for all $i \in N$. Replacing the bids $b_{j}^{i}$ in Mechanism B by $\alpha_{i} b_{j}^{i}, i, j \in N, j \neq i$, yielding the weighted net bids $B_{\alpha}^{j}=$ $\sum_{k \in N \backslash\{j\}}\left(\alpha_{j} b_{k}^{j}-\alpha_{k} b_{j}^{k}\right)$, results in a mechanism that implements in SPE the weighted hierarchical outcome $W_{H}^{\alpha}(N, v, L)$ of a tree game $(N, v, L)$ with $(N, v) \in \mathcal{G}$ zero-monotonic. In Appendix $\mathrm{C}$ we provide some further insight into this statement.

\section{References}

Béal, S., E. Rémila and P. Solal (2010), Rooted-tree solutions for tree games, European Journal of Operational Research, 203, 404-408.

Brink, J.R. van den, and Y. Funaki (2010), Axiomatization and Implementation of Discounted Shapley Values, Tinbergen Institute Discussion Paper 2010-065/1, Tinbergen Institute and VU University, Amsterdam.

Brink, J.R. van den, Y. Funaki, and Y. Ju (2011), Reconciling marginalism with egalitarianism: consistency, monotonicity, and implementation of egalitarian Shapley values, Social Choice and Welfare, doi: 10.1007/s00355-011-0634-2.

Brink, J.R. van den, G. van der Laan and N. Moes (2012), Fair agreements for sharing international rivers with multiple springs and externalities, Journal of Environmental Economics and Management, 63, 388-403.

Demange, G. (2004), On group stability in hierarchies and networks, Journal of Political Economy 112, 754-778.

\footnotetext{
${ }^{9}$ The weighted hierarchical outcomes were introduced by Béal, Rémila and Solal (2010) and van den Brink, van der Laan and Moes (2012) in the context of river games.
} 
Hart, S. and A. Mas-Colell (1989), Potential, value and consistency, Econometrica 57, 589-614.

Herings, P.J.J., G. van der Laan and A.J.J. Talman (2008), The average tree solution for cycle-free graph games, Games and Economic Behavior 62, 77-92.

Herings, P.J.J, G. van der Laan, A.J.J. Talman and Z. Yang (2010), The average tree solution for cooperative games with communication structure, Games and Economic Behavior 68, 626-633.

Ju, Y, and D. Wettstein (2009), Implementing cooperative solution concepts: a generalized bidding approach, Economic Theory 39, 307-330.

Maschler, M. and G. Owen (1989), The consistent Shapley value for hyperplane games, International Journal of Game Theory 18, 389-407.

Macho-Stadler, I., D. Pérez-Castrillo and D. Wettstein (2006), Efficient bidding with externalities, Games and Economic Behavior 57, 304-320.

Mutuswami, S., D. Pérez-Castrillo and D. Wettstein (2004), Bidding for the surplus: realizing efficient outcomes in economic environments, Games and Economic Behavior 48, 111-123.

Myerson, R.B. (1977), Graphs and cooperation in games, Mathematics of Operations Research $2,225-229$.

Pérez-Castrillo, D. and D. Wettstein (2001), Bidding for the surplus: a non-cooperative approach to the Shapley value, Journal of Economic Theory 100, 274-294.

Pérez-Castrillo, D. and D. Wettstein (2002), Choosing wisely: a multi-bidding approach, American Economic Review 92, 1577-1587.

Pérez-Castrillo, D. and D. Wettstein (2005), Forming efficient networks, Economic Letters 87, 83-87.

Shapley, L.S. (1953), A value for $n$-person games, in Contributions to the Theory of Games, Vol. II, ed. H.W. Kuhn and A.W. Tucker, Princeton University Press, Princeton, 307-317.

Slikker, M. (2007), Bidding for surplus in network allocation problems, Journal of Economic Theory 137, 493-511.

Vidal-Puga, J. and G. Bergantiños (2003), An implementation of the Owen value, Games and Economic Behavior 44, 412-427. 


\section{Appendix A}

To describe the mechanism that implements the marginal vector $m^{\pi}(N, v)$ formally, let $N_{t}=$ $N \backslash \cup_{k=1}^{t-1} \rho(k)=\cup_{k=t}^{n} \rho(k)$ be the player set at the start of each round $t, t=1, \ldots, n$. Notice that $N_{t+1}=N_{t} \backslash\{\rho(t)\}$.

Mechanism to implement the marginal vector $m^{\pi}(N, v)$ of a game $(N, v)$.

Initiation: Let $\rho(k)=\pi^{-1}(n+1-k), k \in N$, set $t=1$ and go to Stage 1 of round 1 .

Stage 1: If $t=n$, the mechanism ends and player $\rho(n)$ receives its stand-alone worth $v(\{\rho(n)\})$. If $t<n$, player $\rho(t)$ proposes an offer $y_{j}^{\rho(t)} \in \mathbb{R}$ to every player $j \in N_{t+1}$. Go to Stage 2 .

Stage 2: The players in $N_{t+1}$, sequentially (according to $\rho$ ), either accept or reject the offer. If all players accept, then the proposal is accepted. If at least one player rejects, the proposal is rejected. Go to Stage 3 .

Stage 3: If the proposal is accepted, then each player $j \in N_{t+1}$ receives $y_{j}^{\rho(t)}$, player $\rho(t)$ obtains the remainder $v\left(N_{t}\right)-\sum_{j \in N_{t+1}} y_{j}^{\rho(t)}$ of the payoff in this round and the mechanism ends. If the proposal is rejected, then player $\rho(t)$ leaves the mechanism and obtains its standalone worth $v(\{\rho(t)\})$. The players in $N_{t+1}$ go to the next round to bargain over $v\left(N_{t+1}\right)$. Set $t$ equal to $t+1$ and return to Stage 1 .

This mechanism leads to Theorem 3.1 of Section 3. To give a proof of this theorem we first introduce some notation. Recall from Section 3 that for given ordering $\pi$ on $N, \rho(k)=\pi^{-1}(n+$ $1-k$ ) is the player in $N$ with rank number $n+1-k$ in $\pi, k=1, \ldots, n$, and that for $t=1, \ldots, n, N_{t}=\{\rho(t), \ldots, \rho(n)\}$ is the set of players at the start of round $t$ of the mechanism. For $t=1, \ldots, n$ we further denote $\pi_{t}: N_{t} \rightarrow\{1, \ldots, n+1-t\}$ as $\pi_{t}(k)=\pi(k)$ for all $k \in N_{t}$, $\pi_{t}^{i}=\left\{j \in N_{t} \mid \pi_{t}(j) \leq \pi_{t}(i)\right\}, i \in N_{t}$, and $m_{i}^{\pi_{t}}\left(N_{t}, v_{N_{t}}\right)=v_{N_{t}}\left(\pi_{t}^{i}\right)-v_{N_{t}}\left(\pi_{t}^{i} \backslash\{i\}\right), i \in N_{t}$, i.e., $m_{i}^{\pi_{t}}\left(N_{t}, v_{N_{t}}\right)$ is the marginal vector on the subgame $\left(N_{t}, v_{N_{t}}\right)$ with respect to the ordering $\pi_{t}$. Notice that for $i \in N_{t}, m_{i}^{\pi_{t}}\left(N_{t}, v_{N_{t}}\right)=v_{N_{t}}\left(\pi_{t}^{i}\right)-v_{N_{t}}\left(\pi_{t}^{i} \backslash\{i\}\right)=v\left(\pi_{t}^{i}\right)-v\left(\pi_{t}^{i} \backslash\{i\}\right)=$ $v\left(\pi^{i}\right)-v\left(\pi^{i} \backslash\{i\}\right)=m_{i}^{\pi}(N, v)$, i.e., for every $i \in N_{t}$ the payoff of $i$ in $m^{\pi_{t}}\left(N_{t}, v_{N_{t}}\right)$ is equal to the payoff of $i$ in the marginal vector $m^{\pi}(N, v)$ on the game $(N, v)$.

Proof of Theorem 3.1. (i) We first show that the marginal vector payoffs are indeed equilibrium payoffs of the mechanism. Consider the strategies in which at any round $t<n$ in Stage 1 the proposer $\rho(t)$ offers $y_{j}^{\rho(t)}=m_{j}^{\pi}(N, v)$ to every player $j \in N_{t} \backslash\{\rho(t)\}$ and in Stage 2 the players $j \in N_{t} \backslash\{\rho(t)\}$ accept any offer greater than or equal to $m_{j}^{\pi}(N, v)$ and reject any offer strictly smaller than $m_{j}^{\pi}(N, v)$. These strategies constitute a SPE. Clearly, the strategy at Stage 1 is a best response for the proposer as long as $v\left(N_{t}\right)-\sum_{j \in N_{t} \backslash\{\rho(t)\}} m_{j}^{\pi}(N, v) \geq v(\{\rho(t)\})$. Since $\sum_{j \in N_{t} \backslash\{\rho(t)\}} m_{j}^{\pi}(N, v)=v\left(N_{t} \backslash\{\rho(t)\}\right)$, this holds by definition of zero-monotonicity. Also, at Stage 2 the strategy of every player $j \in N_{t} \backslash\{\rho(t)\}$ is a best response as long as $m_{j}^{\pi}(N, v)=v\left(\pi^{j}\right)-v\left(\pi^{j} \backslash\{j\}\right) \geq v(\{j\})$, which again follows from the definition of zeromonotonicity. 
(ii) Next we show by induction on the number of players that the payoffs in any SPE outcome of the mechanism are equal to the marginal vector payoffs $m_{i}^{\pi}(N, v), i \in N$. For a game $(N, v)$ with $|N|=n=1$ we have that in the first round $t=1=n$ and so the single player $i \in N$ immediately receives its stand-alone worth $v(\{i\})$, which is equal to its marginal vector payoff $m_{i}^{\pi}(N, v)$. Hence, any SPE yields the marginal vector $m^{\pi}(N, v)$.

Now, suppose that for every $\left(N_{k}, v_{N_{k}}\right)$ with $k \geq t+1$, (thus $\left|N_{k}\right|=n+1-k \leq n-t$ ) and given ordering $\pi$, every SPE of the mechanism implements the marginal vector $m_{k}^{\pi}\left(N_{k}, v_{N_{k}}\right)$.

We show that at Stage 2 of round $t$ all players $j \in N \backslash\{\rho(t)\}$ accept the offer if $y_{j}^{\rho(t)}>$ $m_{j}^{\pi}(N, v)$, while the proposal is rejected if $y_{j}^{\rho(t)}<m_{j}^{\pi}(N, v)$ for at least one $j \in N_{t} \backslash\{\rho(t)\}=$ $N_{t+1}$. In case of rejection, player $\rho(t)$ leaves with its own worth and by the induction argument, the payoff to a player $j \in N_{t+1}$ is $m_{j}^{\pi_{t+1}}\left(N_{t+1}, v_{N_{t+1}}\right)=m_{j}^{\pi}(N, v)$. If in Stage 2 of round $t$ the (last) player $\rho(n)$ is reached, its optimal strategy is thus to accept any offer higher than $m_{\rho(n)}^{\pi_{t+1}}\left(N_{t+1}, v_{N_{t+1}}\right)=m_{\rho(n)}^{\pi}(N, v)$ and to reject any offer lower than $m_{\rho(n)}^{\pi}(N, v)$. The second to last player $\rho(n-1)$ anticipates the reaction of player $\rho(n)$. So, if $y_{\rho(n)}^{\rho(t)}>m_{\rho(n)}^{\pi}(N, v)$ and in Stage 2 of round $t$ player $\rho(n-1)$ is reached, then this player accepts the offer if $y_{\rho(n-1)}^{\rho(t)}>m_{\rho(n-1)}^{\pi_{t+1}}\left(N_{t+1}, v_{N_{t+1}}\right)=m_{\rho(n-1)}^{\pi}(N, v)$ and rejects the offer if $y_{\rho(n-1)}^{\rho(t)}<m_{\rho(n-1)}^{\pi}(N, v)$. If $y_{\rho(n)}^{\rho(t)}<m_{\rho(n)}^{\pi}(N, v)$, then player $\rho(n-1)$ is indifferent between accepting or rejecting any offer $y_{\rho(n-1)}^{\rho(t)}$, because player $\rho(n)$ is going to reject the offer $y_{\rho(n)}^{\rho(t)}$ anyway. By backwards induction it follows that for every $t=1, \ldots, n-1$ and every SPE, at Stage 2 of round $t$ all players $j \in N_{t+1}$ accept the offer of player $\rho(t)$ if $y_{j}^{\rho(t)}>m_{j}^{\pi}(N, v)$ and that the offer is rejected if $y_{j}^{\rho(t)}<m_{j}^{\pi}(N, v)$ for at least one $j \in N_{t+1}$.

For a round $t$, we now consider two cases. First, if $v\left(N_{t}\right)>v\left(N_{t+1}\right)+v(\{\rho(t)\})$, it follows that the strategies described in the first part of the proof are the only SPE strategies in the subgame that starts at Stage 1 of round $t$. To see this notice that in this case rejection of the proposal made by player $\rho(t)$ can not be part of a SPE because then player $\rho(t)$ would receive $v(\{\rho(t)\})$. The proposer can improve its payoff by taking $0<\epsilon<v\left(N_{t}\right)-v\left(N_{t+1}\right)-v(\{\rho(t)\})$ and offering $m_{j}^{\pi_{t}}\left(N_{t}, v_{N_{t}}\right)+\frac{\epsilon}{\left|N_{t}\right|-1}>m_{j}^{\pi}(N, v)$ to every $j \in N_{t+1}$. Hence, a SPE requires acceptance of the offers in Stage 2. Since any proposal with $y_{j}^{\rho(t)}<m_{j}^{\pi}(N, v)$ for some $j \in N_{t+1}$ is rejected, a SPE requires that $y_{j}^{\rho(t)} \geq m_{j}^{\pi}(N, v)$ for all $j \in N_{t+1}$. On the other hand, any proposal such that $y_{h}^{\rho(t)}>m_{h}^{\pi}(N, v)$ for some $h \in N_{t+1}$ can not be part of a SPE, because then $\rho(t)$ could improve by taking $0<\epsilon<y_{h}^{\rho(t)}-m_{h}^{\pi}(N, v)$ and offering $m_{j}^{\pi}(N, v)+\frac{\epsilon}{\left|N_{t}\right|-1}>m_{j}^{\pi}(N, v)$ to every $j \in N_{t+1}$. It can be concluded that at any SPE in the subgame that starts in round $t$ it must hold that $y_{j}^{\rho(t)}=m_{j}^{\pi}(N, v)$ for all $j \in N_{t+1}$ and that these offers are accepted.

Second we consider the case that $v\left(N_{t}\right)=v\left(N_{t+1}\right)+v(\{\rho(t)\})$ and thus $m_{\rho(t)}^{\pi}=v\left(N_{t}\right)-$ $v\left(N_{t+1}\right)=v(\{\rho(t)\})$. As in the previous case, the strategies described in the first part of the proof are SPE strategies in the subgame that starts at Stage 1 of round $t$. In addition, also any strategy profile in which at Stage 1 of round $t$, player $\rho(t)$ offers $y_{j}^{\rho(t)} \leq m_{j}^{\pi}(N, v)$ to some players $j \in N_{t+1}$ and, at Stage 2, these players $j$ reject any offer $y_{j}^{\rho(t)} \leq m_{j}^{\pi}(N, v)$, constitutes a SPE. In this SPE the proposer receives it own worth $v(\{\rho(t)\})$. 
In both cases it follows that every player $j \in N_{t}$ receives $m_{j}^{\pi}(N, v)$ in every $\mathrm{SPE}$ of the subgame that starts in round $t$. So, every SPE of the mechanism results in payoff vector $m^{\pi}(N, v)$.

\section{Appendix B}

Proof of Theorem 3.2. First, it follows straightforwardly that for every tree $(N, L)$ the restricted game $\left(N, v^{L}\right)$ is zero-monotonic if $(N, v)$ is zero-monotonic. Second, as discussed in Section 2, for given player $i \in N$, the hierarchical outcome $h^{i}(N, v, L)$ is equal to the marginal vector $m^{\pi}\left(N, v^{L}\right)$ of the restricted game $\left(N, v^{L}\right)$ for every ordering $\pi$ that is consistent with the rooted directed tree $\left(N, L^{i}\right)$. Notice that there can be multiple ordering $\pi \in \Pi(N)$ satisfying these conditions, but that for every such $\pi$ it holds that $h^{i}(N, v, L)=m^{\pi}\left(N, v^{L}\right)$. Further, since every $j \neq i$ is a subordinate of $i$ we have that for every such $\pi$ it holds that $\pi(i)=n$. According to Mechanism A, $i$ is the proposer in the first round.

To show that the payoffs in the hierarchical outcome $h^{i}(N, v, L)$ are indeed the payoffs of Mechanism A in every SPE, consider the following strategies. In every round $t \geq 1$, every proposer $j \in P^{t}$ proposes in Stage 1 the hierarchical outcome payoff $y_{k}^{j}=h_{k}^{i}(N, v, L)$ to every $k$ in its set of subordinates $S_{j}^{i} \backslash\{j\}$. In Stage 2 every subordinate $k$ of a proposer $j$ accepts any offer at least equal to $h_{k}^{i}(N, v, L)$ and rejects any offer strictly smaller than $h_{k}^{i}(N, v, L)$. Clearly, under these strategies the mechanism ends in round 1 and every player $k \in N$ receives payoff $h_{k}^{i}(N, v, L)$, being the payoff of the marginal vector $m^{\pi}\left(N, v^{L}\right)$ for an ordering $\pi$ consistent with $\left(N, L^{i}\right)$. The proof that these strategies form an SPE follows from Theorem 3.1. In fact, let $N_{t}$ be the set of players in round $t$. Then the subgraph $\left(N_{t}, L\left(N_{t}\right)\right)$ consists of several components, but on each of these components the rooted tree $\left(N, L^{i}\right)$ induces a rooted subtree. Then in round $t$ on each component the root proposes the hierarchical outcome to each of its subordinates, and these payoffs are equal to the payoffs of these players in every marginal vector $m^{\pi}\left(N, v^{L}\right)$ with $\pi$ consistent with $\left(N, L^{i}\right)$. So, Mechanism A is identical to the mechanism of Theorem 3.1 for every $\pi$ consistent with $\left(N, L^{i}\right)$, except that in a round $t$ there might be multiple proposers, each proposing the hierarchical outcome on its own subtree. Because the mechanism of Theorem 3.1 on a zero-monotonic game $(N, v)$ with initiation $\pi$ implements the marginal vector $m^{\pi}(N, v)$ in SPE, it follows that Mechanism A with initiation $i$ implements in SPE the marginal vector $m^{\pi}\left(N, v^{L}\right)$ for every $\pi$ consistent with $\left(N, L^{i}\right)$, i.e. $h^{i}(N, v, L)$.

\section{Appendix C}

In Section 4 it is stated that given a vector of weights $\alpha \in \mathbb{R}_{+}^{N}$ with $\sum_{i \in N} \alpha_{i}=1$, there exists a mechanism that implements in SPE the weighted hierarchical outcome $\operatorname{WHO}^{\alpha}(N, v, L)$ of a tree game $(N, v, L)$ with $(N, v) \in \mathcal{G}$ zero-monotonic. 
Mechanism $\mathbf{C}$ to implement the weighted hierarchical outcome $\operatorname{WHO}^{\alpha}(N, v, L)$ of a tree game $(N, v, L)$. This mechanism is the same as Mechanism $\mathrm{B}$, except that the net bid $B^{j}, j \in N$, is replaced by the weighted net bid $B_{\alpha}^{j}=\sum_{k \in N \backslash\{j\}}\left(\alpha_{j} b_{k}^{j}-\alpha_{k} b_{j}^{k}\right)$.

Lemma 4.1 of Section 4 now changes to the following lemma.

Lemma 4.3 Let $(N, v, L) \in \mathcal{G} \mathcal{L}$ be a tree game with $(N, v)$ zero-monotonic. Given the vector of weights $\alpha \in \mathbb{R}_{+}^{N}$ with $\sum_{i \in N} \alpha_{i}=1$, let $b_{j}^{i}=W_{H} O_{j}^{\alpha}(N, v, L)-h_{j}^{i}(N, v L)$. Then, for every $i \in N, B_{\alpha}^{i}=0$.

Proof. For every $i \in N$ we have that

$$
\begin{aligned}
B_{\alpha}^{i} & =\sum_{j \in N \backslash\{i\}}\left(\alpha_{i} W H O_{j}^{\alpha}(N, v, L)-\alpha_{i} h_{j}^{i}(N, v, L)-\alpha_{j} W H O_{i}^{\alpha}(N, v, L)+\alpha_{j} h_{i}^{j}(N, v, L)\right) \\
& =\alpha_{i} \sum_{j \in N \backslash\{i\}} W H O_{j}^{\alpha}(N, v, L)-\left(1-\alpha_{i}\right) W H O_{i}^{\alpha}(N, v, L)+\sum_{j \in N \backslash\{i\}}\left(\alpha_{j} h_{i}^{j}(N, v, L)-\alpha_{i} h_{j}^{i}(N, v, L)\right) \\
& =\alpha_{i} \sum_{j \in N} W H O_{j}^{\alpha}(N, v, L)-W H O_{i}^{\alpha}(N, v, L)+\sum_{j \in N \backslash\{i\}}\left(\alpha_{j} h_{i}^{j}(N, v, L)-\alpha_{i} h_{j}^{i}(N, v, L)\right) \\
& =\alpha_{i} v(N)-\sum_{k \in N} \alpha_{k} h_{i}^{k}(N, v, L)+\sum_{j \in N \backslash\{i\}}\left(\alpha_{j} h_{i}^{j}(N, v, L)-\alpha_{i} h_{j}^{i}(N, v, L)\right) \\
& =\alpha_{i} v(N)-\alpha_{i} \sum_{k \in N} h_{k}^{i}(N, v, L)=0,
\end{aligned}
$$

where the second equality follows from the fact that $\alpha_{i}+\sum_{j \in N \backslash\{i\}} \alpha_{j}=1$, the fourth from efficiency of the weighted hierarchical outcomes and the last from efficiency of the hierarchical outcomes.

With the help of this lemma the proof of the next theorem follows in the same way as the proof of Theorem 4.2 and is therefore omitted.

Theorem 4.4 Let $(N, v, L) \in \mathcal{G} \mathcal{L}$ be a tree game with $(N, v)$ zero-monotonic and let $\alpha \in \mathbb{R}_{+}^{N}$ with $\sum_{i \in N} \alpha_{i}=1$ be a vector of weights. Then the payoff vector in any SPE of Mechanism $C$ coincides with the payoff vector $W_{H} H^{\alpha}(N, v, L)$.

It holds that Mechanism $\mathrm{C}$ has a unique $\mathrm{SPE}$ when $(N, v) \in \mathcal{G}$ is strictly zero-monotonic. In that case each player $k \in N$ makes bids $b_{j}^{k}=W_{H} O_{j}^{\alpha}(N, v, L)-h_{j}^{k}(N, v, L)$ to the players $j \in N \backslash\{k\}$ in the bidding procedure; some player $i$ is randomly chosen to be the winner and so is the proposer in round 1 of the proposal procedure. This player offers $y_{j}^{i}=h_{j}^{i}(N, v, L)$ to every player $j \in N \backslash\{i\}$ and every player $j \in N \backslash\{i\}$ accepts the offer. 